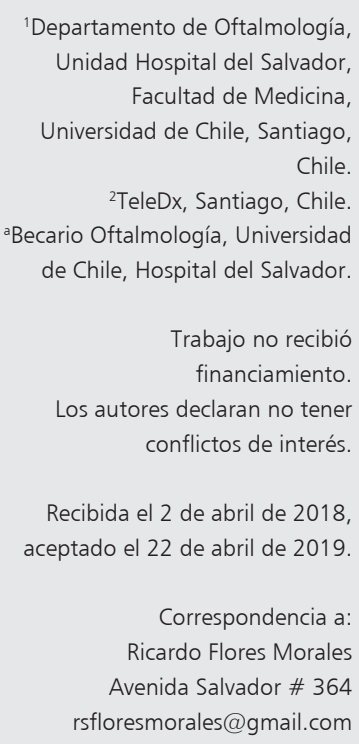

Correspondencia a: Ricardo Flores Morales Avenida Salvador \# 364 rsfloresmorales@gmail.com

\section{Modelo de manejo en red y por telemedicina de la retinopatía diabética en dos comunas del Servicio de Salud Metropolitano Oriente}

\author{
RICARDO FLORES-MORALES ${ }^{1, \mathrm{a},}$, RODRIGO DONOSO ${ }^{1}$, \\ JOSÉ TOMÁS ARENAS-CAVALLI ${ }^{2}$, RODRIGO ANGUITA ${ }^{1}$
}

\section{Management of diabetic retinopathy using telemedicine and network integration}

Background: If we are able to increase the resolution of complex medical problems at primary health care levels, we would improve the efficiency of the health care systems and would reduce the burden of specialists. Aim: To describe the result of a telemedicine and network management of diabetic retinopathy supervised by an ophthalmological service. Material and Methods: Diabetic patients attended in primary health care centers of the East Metropolitan Health Service in Santiago, Chile, derived for $45^{\circ}$ digital retinographies were evaluated using telemedicine by the ophthalmologists of the base hospital. These professionals screened for diabetic retinopathy and classified it. Those patients with diabetic retinopathy were derived to the base hospital for specialized management. Results: Of 2,566 patients evaluated, 22\% had signs of diabetic retinopathy, 75\% did not have the condition and $2 \%$ could not be evaluated with retinography. Seventy percent of patients with retinopathy, equivalent to only fifteen percent of total diabetics, were referred to the specialists for treatment. Conclusions: This model allowed a reduction of referrals to ophthalmologists, reducing the burden of secondary and tertiary health care systems.

(Rev Med Chile 2019; 147: 444-450)

Key words: Diabetic Retinopathy; Primary Health Care; Telemedicine.
$\mathrm{L}$ a diabetes mellitus (DM) es una pandemia que, según cifras publicadas por la Organización Mundial de la Salud (OMS) y la Federación Internacional de Diabetes (FID), está en constante crecimiento, estimándose que para 2040 existirán cerca de 642 millones de diabéticos en el mundo ${ }^{1}$. Se estima que en 2014 había 62 millones de personas con DM en la región de las Américas, lo que supone uno de cada 12 habitantes. Específicamente, en América Central y América del Sur en el año 2015 había 29,6 millones de personas con diabetes y se proyecta que serán 48,8 millones en 2040, lo que representa uno de los mayores incrementos de prevalencia del mundo ${ }^{1}$. Sin duda, la DM es un problema crítico de salud pública a nivel mundial, y así lo manifiesta la $\mathrm{OMS}^{2}$. La importancia epidemiológica de la DM no depende exclusivamente de su prevalencia, sino también de la carga de morbilidad y mortalidad que trae asociada, con el consecuente impacto social y económico. En un estudio de corte transversal, $27,8 \%$ de una muestra de pacientes diabéticos tenía enfermedad renal, 18,9\% daño ocular y $9 \%$ enfermedad coronaria ${ }^{3}$.

En Chile, según datos entregados por la Encuesta Nacional de Salud (ENS), en el año 2003 había una prevalencia de DM en mayores de 15 años de 6,3\%, que aumentó a 9,4\% el año 2010, resultando en un incremento de $49 \%$ en 7 años ${ }^{4,5}$, y sigue aumentando puesto que, según la FID, en 
el 2015 había 1.372 .700 personas con DM, lo que representa a $11 \%$ de la población adulta entre 20 y 79 años $^{6}$. Esta cifra ubica a nuestro país en el segundo lugar de Sudamérica.

La retinopatía diabética (RD), tercera causa de ceguera en el mundo se ha convertido en la primera causa de ceguera en la población laboralmente activa en occidente $e^{7,8}$ y más de $30 \%$ de los diabéticos tiene un déficit visual por RD (3,7 millones en el mundo $)^{9}$. Múltiples estudios reportan cifras comprendidas entre $20 \%$ y $50 \%$ de $\mathrm{RD}$ en diabéticos ${ }^{10}$. Investigaciones informan que al momento del diagnóstico de DM2, 15-20\% presentaba RD, cifra que se eleva a $35-40 \%$ a los 10 años de enfermedad, $80 \%$ a los 15 años y cercana a $100 \%$ a los 20 años ${ }^{11}$.

Un adecuado control de la diabetes y el tamizaje sistemático para detectar y tratar precozmente la $\mathrm{RD}$, mediante fotocoagulación, son las medidas más efectivas para la prevención de la pérdida visual y ceguera causada por diabetes ${ }^{12}$. Pero como la población diabética sigue aumentando ${ }^{13}$, el tamizaje se hace cada vez más insuficiente. Incluso en países desarrollados, la cobertura del examen de fondo de ojo no alcanza a $50 \%$ de los pacientes diabéticos ${ }^{14}$.

En los países en vías de desarrollo, la cobertura del tamizaje de los pacientes diabéticos definitivamente no es suficiente para prevenir sus complicaciones, lo que se traduce en pérdida visual grave, ceguera, mayores costos en su tratamiento, además del impacto social de esta población laboralmente activa $^{6,15}$. En Chile, la población diabética fue de alrededor de $11 \%$ en $2015^{6}$ y su cobertura de tamizaje de RD no fue más de $15 \%{ }^{16}$.

Esta problemática sigue siendo desafío de salud pública en todo el mundo, debido a la carencia relativa de recursos oftalmológicos, oftalmólogos y profesionales capaces de examinar el fondo de ojo o retinografías de estos pacientes en permanente riesgo de pérdida visual y ceguera por su diabetes.

La retinografía digital está bien establecida como método estándar y costo-efectivo de tamizaje para la detección de RD desde el año $2003^{17}$. Muchos países están utilizando dos imágenes digitales de campos estandarizados, las que son analizadas por un profesional especializado, directamente o a través de telemedicina, para informar y clasificar la $\mathrm{RD}^{18,19}$. En el Reino Unido, 1,7 millones de personas con diabetes fueron sometidas a tamizaje de $\mathrm{RD}$ con este método entre 2007 y $2008^{20}$. Junto a Holanda y Escocia, fueron los primeros países en implementar un proyecto de tamizaje en sus programas nacionales de RD en la última década con excelentes resultados, tanto que recientemente dejó de ser la primera causa de ceguera en personas en edad laboral en el Reino Unido ${ }^{21}$.

En Chile, como en muchos otros países en vías de desarrollo, en los programas de salud pública los recursos oftalmológicos también son insuficientes para el tamizaje anual de todos los pacientes diabéticos (11\% de la población). Por ello, nuestro propósito es describir un innovador modelo de manejo en red y por telemedicina de la $\mathrm{RD}$, desde su tamizaje a nivel primario hasta su resolución terciaria en Departamento de Retina. Esto, bajo normas, supervisión y atención del Servicio de Oftalmología del Hospital del Salvador, en el Servicio de Salud Metropolitano Oriente (SSMO), que es el establecimiento que finalmente atiende y trata sus complicaciones.

\section{Pacientes y Métodos}

Estudio descriptivo retrospectivo de la implementación, durante 4 meses de 2016, de un modelo de manejo en red y por telemedicina de la RD normado, supervisado y atendido por el Servicio de Oftalmología del Hospital del Salvador (HDS), desde el tamizaje a nivel primario hasta su resolución terciaria a nivel hospitalario.

Este modelo se inicia en la Atención Primaria de Salud (APS) de las comunas de Nuñoa y Peñalolén, pertenecientes al SSMO, desde donde se derivaron la totalidad de los pacientes diabéticos en control que requerían fondo de ojo a las respectivas unidades de atención primaria oftalmológica (UAPO) para la toma de 2 retinografías digitales estándar de $45^{\circ}$ por cada ojo, por un tecnólogo médico con mención en Oftalmología entrenado, obteniendo 4 imágenes por paciente, una de campo temporal con mácula y la otra de campo nasal con disco óptico, por cada ojo. Ello, usando una cámara de retina no midriática, Canon CR-DGI (EOS 30D) (Canon Inc., Tokio, Japón).

Las imágenes fueron reducidas a 701 x 468 píxeles y registradas secuencialmente en forma numérica, según el orden de realización, para luego enviarlas mediante telemedicina a oftalmólogos del Servicio de Oftalmología del HDS, quienes hacían el tamizaje de acuerdo con la presencia 
o ausencia de RD y la clasificación clásica en etapas, según la severidad de esta. Se seleccionaron aquellas retinografías con signos de RD y se correlacionaron según el número de registro con los pacientes respectivos, quienes eran derivados al Servicio de Oftalmología para la confirmación diagnóstica por oftalmólogo, mediante el examen del fondo de ojo con oftalmoscopía indirecta. Luego, los pacientes $\sin \mathrm{RD}$ y con $\mathrm{RD}$ no proliferativa (RDNP) leve, volvían a su control en APS y a la UAPO respectivas. En cambio, los diabéticos con un estado más avanzado de RD o cualquier grado de maculopatía eran derivados para su seguimiento y manejo definitivo en el Departamento de Retina del HDS. Aquellas retinografías que no eran posible ser evaluadas por opacidad de medios o mala calidad, también se identificaron con un número de registro para derivarlos al Departamento de Retina para su correcta evaluación diagnóstica y manejo. Por último, aquellas retinografías sin signos de RD, pero con signos de otra patología, eran identificados para ser evaluados por el oftalmólogo en la misma UAPO y si era necesario se derivaban al departamento correspondiente en el HDS, para optimizar su diagnóstico y manejo (Figura 1).

Del total de diabéticos se registraron datos demográficos, tales como: edad, género y centro de salud familiar (CESFAM) de procedencia. Además, se calculó el porcentaje de los diabéticos que tenían algún signo de RD en la retinografía, aquellos sin signos RD y los que no eran evaluables por medio de retinografías. Luego, se calculó el porcentaje de pacientes con signos de $\mathrm{RD}$ que fueron derivados para su manejo a nivel terciario y de aquellos que no fueron derivados, siguiendo en control en UAPO. Por último, del total de diabéticos evaluados en APS, se calculó el porcentaje de pacientes que siguieron en control en las UAPO y de aquellos que se derivaron a nivel terciario.

El estudio se realizó siguiendo los principios de la Declaración de Helsinki, además de obtenerse la aprobación de la Junta de Revisión Local y se estableció un acuerdo con la institución. Debido a la naturaleza anónima de los datos del estudio, ya que no afecta los cuidados normales de los pacientes, el Comité de Ética Institucional del Departamento de Salud Comunal correspondiente, aprobó el estudio y determinó que el consentimiento informado no era necesario.

\section{Resultados}

Se evaluaron con retinografía 2.566 diabéticos en control médico en APS, provenientes de las comunas de Nuñoa y Peñalolén de la Región Metropolitana, durante 4 meses del año 2016. Se evaluaron 358 diabéticos provenientes de 2 Centros de Salud Familiar (CESFAM) de Ñuñoa y 2.208 provenientes de 6 CESFAM de Peñalolén. La edad promedio del total de pacientes fue 63,3 (1598 ) años, 60,2\% eran de género femenino y $39,8 \%$ masculino. La edad promedio y distribución por género de cada CESFAM se muestran en la Tabla 1.

De los 2.566, $1.938(75,5 \%)$ no presentaban signos de RD, 575 (22,4\%) tenían algún signo de RD y $53(2,1 \%)$ no eran evaluables por medio de retinografías (Figura 2). De los 575 pacientes con signos de RD, 158 (27,4\%) tenían RDNP leve, 348 $(60,5 \%)$ RDNP moderada, 51 (8,9\%) RDNP severa y $18(3,2 \%)$ RD proliferativa; 403 (70,1\%) fueron derivados a nivel terciario y 172 (29,9\%) permanecieron en control en las UAPO (Figura 3). Del total de pacientes diabéticos evaluados con retingrafía, $403(15,1 \%)$ fueron derivados para su manejo a nivel terciario en el Departamento de Retina del HDS por presentar RDNP moderada o un estado más avanzado y los demás $2.163(84,9 \%)$ pacientes siguieron en controles en la UAPO (Figura 4). El edema macular diabético clínicamente significativo (EMDCS) se consideró como patología asociada y 100\% de los pacientes con signos sugerentes de EMDCS en la retinografía, además, presentaban un estado avanzado de $\mathrm{RD}$, por el cual fueron derivados para su tratamiento a nivel terciario.

En la evaluación de las retinografías, se detectaron signos sugerentes de otras patologías en 414 diabéticos (16,1\% del total), de los cuales 137 presentaban RDNP moderada o un estadio más avanzado, siendo derivados por esta causa al Departamento de Retina del HDS y el resto, 277 pacientes, fue igualmente derivado por las otras patologías detectadas a los diferentes departamentos del Servicio de Oftalmología del HDS. En la Tabla 2 se describe la frecuencia de las diferentes patologías asociadas que se detectaron.

En suma, del total de diabéticos en control en APS, se derivaron $680(26,5 \%)$ para manejo a nivel terciario, ya sea por RD u otra patología asociada, y $1.886(73,5 \%)$ siguieron en controles en APS y las UAPO por oftalmólogos, según las normas del Servicio de Oftalmología del hospital. 
Manejo en red y por telemedicina de la retinopatía diabética - R. Flores et al

Tabla 1. Cantidad, edad promedio y distribución porcentual por género de los diabéticos en control en los diferentes Centros de Salud Familiar (CESFAM) de la comuna de Nuñoa y Peñalolén que fueron evaluados por retinografías

\begin{tabular}{|llcccc|}
\hline Comuna & CESFAM & Diabéticos & Edad promedio* & Masculino & Femenino \\
Peñalolén & Carol Urzúa & 502 & $63,9(19-98)$ & $196(39 \%)$ & $306(61 \%)$ \\
& San Luis & 561 & $63,6(17-94)$ & $240(42,7 \%)$ & $321(57,3 \%)$ \\
& Padre Gerardo Whelan & 378 & $62,1(92-34)$ & $139(36,7 \%)$ & $239(63,3 \%)$ \\
& La Faena & 312 & $62,3(18-92)$ & $121(38,7 \%)$ & $191(61,3 \%)$ \\
& Lo Hermida & 335 & $62,6(21-90)$ & $156(46,5 \%)$ & $179(53,5 \%)$ \\
& Cardenal Silva Henríquez & 243 & $62,8(15-95)$ & $82(33,7 \%)$ & $161(66,3 \%)$ \\
\multirow{2}{*}{ Nuñoa } & Rosita Renard & 118 & $66,9(31-94)$ & $47(39,8 \%)$ & $71(60,2 \%)$ \\
& Salvador Bustos & 117 & $66,7(35-97)$ & $42(35,8 \%)$ & $75(64,2 \%)$ \\
\hline
\end{tabular}

*Se expresa en años y entre paréntesis el rango etario.
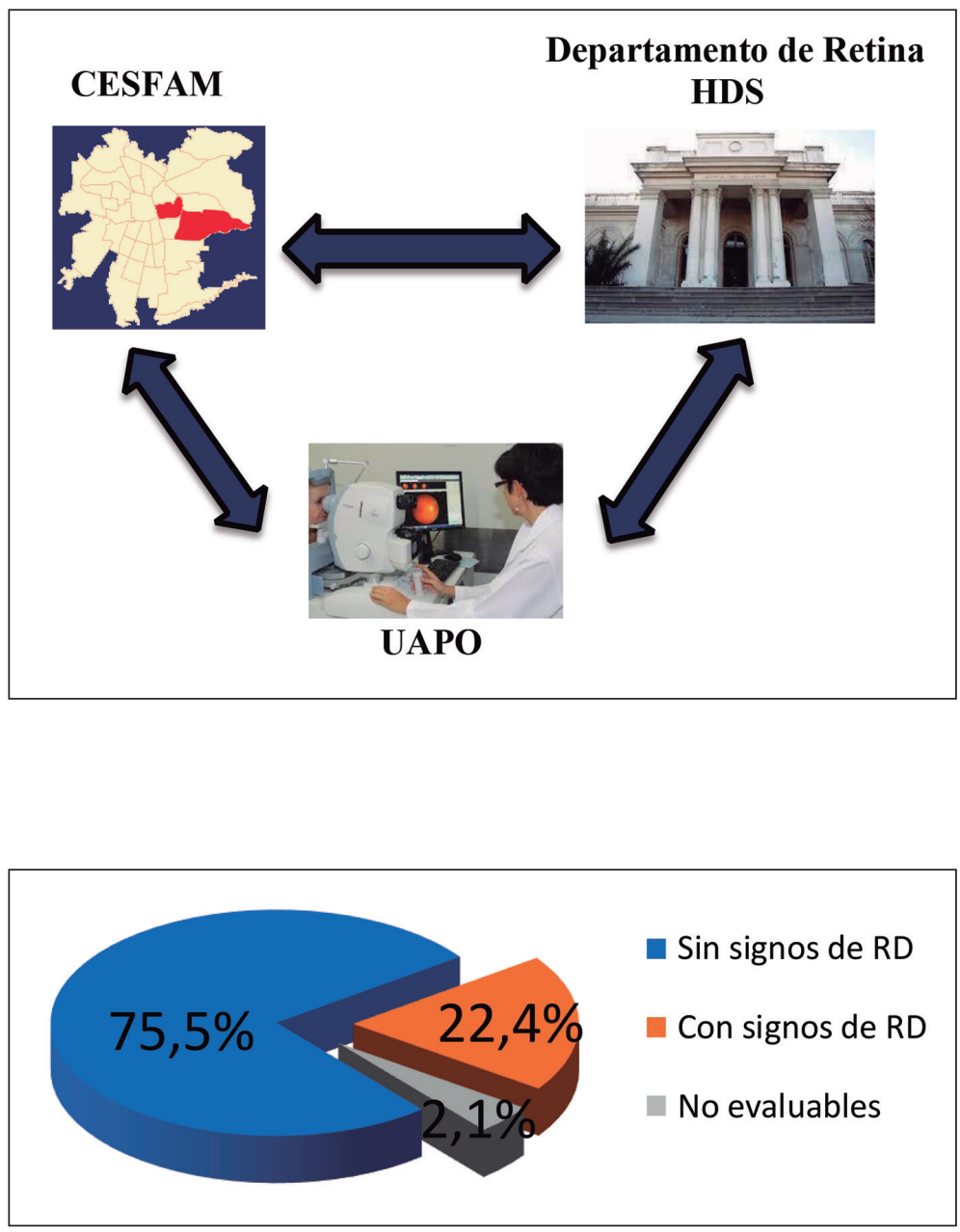

Figura 1. Representación esquemática de la red de atención oftalmológica para la retinopatía diabética. Arriba a la izquierda se representa la atención primaria de salud por medio de los centros de salud familiar (CESFAM) de 2 comunas del Servicio de Salud Metropolitano Oriente, Abajo al centro el nivel secundario de salud por medio de las unidades de atención primaria oftalmológica (UAPO) y arriba a la derecha el nivel terciario por medio del Departamento de Retina del Hospital del Salvador (HDS).
Figura 2. Diabéticos con signos de RD, sin signos de RD y no evaluables. Gráfico que muestra el porcentaje del total de diabeticos evaluados, que tenía signos de RD, sin signos de RD y aquellos no evaluables por retinografía. 


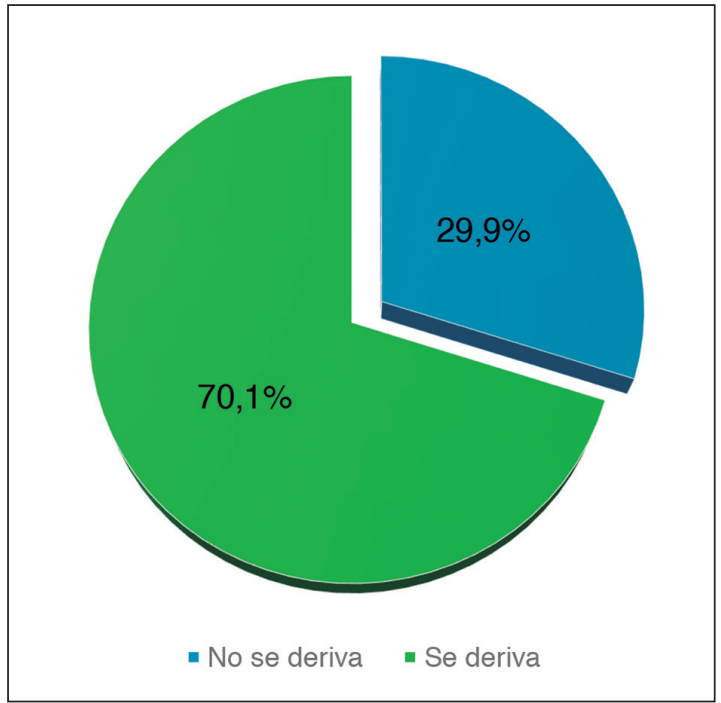

Figura 3. Diabéticos con signos de RD, controlados en UAPO y derivados a nivel terciario. Gráfico que muestra el porcentaje de diabéticos con signos de RD en la retinografía, que se controlaron en las unidades de atención primaria oftalmológica y aquellos que se derivaron a nivel terciario.

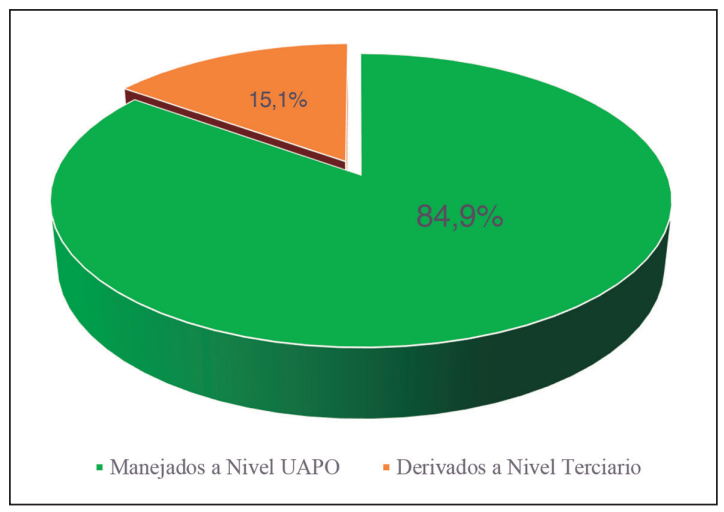

Figura 4. Total de diabéticos de APS, controlados en UAPO y derivados a nivel terciario. Gráfico, que muestra el porcentaje del total de diabéticos controlados en Atención Primaria de Salud (APS), que se manejaron a nivel de las unidades de atención primaria oftalmológica (UAPO) y aquellos que se manejaron a nivel terciario.

\section{Discusión}

La prevalencia de DM está creciendo en todo el mundo y la única manera de prevenir la disminución visual por $\mathrm{RD}$ es mediante un ta-
Tabla 2. Frecuencia de las diferentes patologías asociadas que se pesquisaron, a través de la evaluación por telemedicina de las retinografías

\begin{tabular}{|cc|}
\hline Patologías asociadas & Pacientes \\
\hline Catarata & 125 \\
\hline Maculopatía N/E & 117 \\
\hline Glaucoma & 62 \\
\hline EMDCS & 23 \\
\hline DMRE & 15 \\
\hline Fibrosis preretinal & 12 \\
\hline Alta ametropias & 9 \\
\hline Otra & 51 \\
\hline
\end{tabular}

(N/E: no especificada, EMDCS: edema macular diabético clinicamente significativo según clasificación del ETDRS, DMRE: degeneración macular relacionada a la edad).

mizaje anual ${ }^{22}$. Los recursos oftalmológicos para el tamizaje establecido con el fin de lograr una detección y tratamiento temprano de $\mathrm{RD}$ serán más insuficientes cada año debido al crecimiento descontrolado de su prevalencia ${ }^{23}$.

Por otra parte, el uso de mejores imágenes digitales y su transmisión a distancia ha aumentado, facilitado por tecnología computacional más asequible en centros de atención ocular primaria en los países desarrollados y en desarrollo. Esto ha hecho que los exámenes, informes y referencias por telemedicina sean más oportunos y costo efectivo para el manejo oftalmológico durante los últimos años ${ }^{24}$, estando validado el tamizaje de RD por retinografía digital desde el año $2003^{17}$.

En los últimos años, se han desarrollado softwares que realizan tamizaje automatizado de retinografías mediante inteligencia artificial, como solución para aumentar su cobertura ante la creciente falencia relativa del recurso oftalmológico. Softwares que están en evaluación en distintos sistemas de salud, como en el Reino Unido, India, Singapur, Canadá, etc. ${ }^{25-28}$. En Chile se ha desarrollado y validado en forma pionera un software de diagnóstico automatizado de retinografías telemáticas (DART), el cual se estaba aplicando como piloto desde 2017 en las comunas de Ñuñoa y Peñalolén, apoyando esta iniciativa de manejo en red de la RD en el SSMO; implementándose en forma oficial desde mayo de 2018 en 28 CESFAM del país ${ }^{29}$. 
Tal iniciativa ayuda a identificar a los pacientes con presencia de cualquier grado de RD (a nivel de umbral de referencia o en riesgo de pérdida visual), liberando del examen oftalmológico especializado a aquellos pacientes con retinografía normal o con incipientes lesiones de RD y dejando el escaso recurso oftalmológico disponible para el manejo y tratamiento de los casos más avanzados o complicados, aumentando de manera importante la eficiencia tanto del recurso oftalmológico como financiero para los programas de prevención en pacientes diabéticos.

Tanto en Chile como en otros países más desarrollados, estos softwares de detección automatizada de RD están validándose y siendo aceptados por las autoridades sanitarias para su aplicación como parte de los programas nacionales de tamizaje de $\mathrm{RD}^{16-19,25}$.

Por el momento, y de acuerdo a nuestros resultados, hacen razonable nuestra propuesta de un modelo de tamizaje por telemedicina y manejo en red desde el servicio de oftalmología del hospital, que racionalice, mejore y haga más eficiente la prevención y manejo de $\mathrm{RD}$, en esta cada vez más prevalente enfermedad.

La aplicación de esta atención en red en dos comunas de la Región Metropolitana, durante 4 meses del año 2016, permitió que solo 15,1\% de los diabéticos atendidos a nivel primario fueron derivados a atención terciaria y $84,9 \%$ restante se resolvió a nivel de la UAPO en coordinación con la APS, cifras que avalan el impacto positivo de este modelo en la efectividad del tamizaje y manejo de $\mathrm{RD}$ en nuestro país, permitiendo no solo aumentar la cobertura de este, sino también concentrar los limitados recursos de oftalmólogos especialistas en retina para el tratamiento de los pacientes con RD más avanzada.

También es interesante mencionar que, mediante este modelo, se logró pesquisar y manejar adecuadamente, en 16,1\% de la población evaluada, otras patologías oftalmológicas coexistentes que potencialmente también pueden generar déficit visual y ceguera en estos pacientes diabéticos.

En conclusión, de la implementación de este modelo de manejo en red y por telemedicina de la $\mathrm{RD}$ destaca que un bajo porcentaje de pacientes con RD de estas 2 comunas del SSMO fueron derivados a nivel terciario, permitiendo racionalizar eficientemente el recurso oftalmológico especializado para el tratamiento de RD más avanzada.
Haciendo énfasis en que es necesario más esfuerzos como este, con el apoyo de la tecnología, la inteligencia artificial y la atención en red, para poder construir e implementar en el sistema público de salud un modelo de atencion de la RD eficiente y eficaz, que permita mejorar la salud visual de los pacientes diabéticos y prevenir una importante causa de ceguera y discapacidad visual, sobre todo en la población laboralmente activa.

\section{Referencias}

1. International Diabetes Federation 2015. Disponible en: www.idf.org/diabetesatlas [Consultado el 01 de abril de 2018].

2. Día mundial de la salud, 2016. Disponible en: www. minsal.cl [Consultado el 01 de abril de 2018].

3. Danaei G, Finucane MM, Lu Y, Singh GM, Cowan MJ, National, regional, and global trends in fasting plasma glucose and diabetes prevalence since 1980: systematic analysis of health examination surveys and epidemiological studies with 370 country-years and 2.7 million participants. Lancet 2011; 378 (9785): 31-40.

4. Encuesta Nacional de Salud 2003(ENS). Disponible en: www.minsal.cl [Consultado el 01 de abril de 2018].

5. Encuesta Nacional de Salud 2009-2010 (ENS). Disponible en: www.minsal.cl [Consultado el 01 de abril de 2018].

6. Fleming AD, Goatman KA, Philip S, Williams GJ, Prescott GJ, Scotland GS, et al. The role of haemorrhage and exudate detection in automated grading of diabetic retinopathy. Scottish Diabetic Retinopathy Clinical Research Network. Br J Ophthalmol 2010; 94 (6): 706-11.

7. Barría F, Martínez F. Guía Práctica clínica de retinopatía diabética para Latinoamérica. The International Agency for the Prevention of Blindness. 2011.

8. Kocur I, Resnikoff S. Visual impairment and blindness in Europe and their prevention. Br J Ophthalmol 2002; 86 (7): 716-22.

9. Klein BE. Overview of epidemiologic studies of diabetic retinopathy. Ophthalmic Epidemiol 2007; 14: 179-83.

10. Méndez T, López R, Rosales C, Seuc A. Prevalencia de retinopatía diabética en un área de salud del municipio Marianao. Rev Cubana Oftalmol 1996; 9 (2): 116-21.

11. Durruty P, Carpentier C, Krause P, García de los Ríos M. Evaluación del compromiso retinal en diabéticos tipo 2 microalbuminúricos. Rev Med Chile 2000; 128: 1085-92.

12. Wilson A, Baker R, Thompson J, Grimshaw G. Coverage in screening for diabetic retinopathy according to 
screening provision: results from a national survey in England and Wales. Diabet Med 2004; 21 (3): 271-8.

13. International Diabetes Federation. IDF Diabetes Atlas update poster, 6th edn. Brussels, Belgium: International Diabetes Federation, 2014.

14. Abràmoff $\mathrm{MD}$, Niemeijer $\mathrm{M}$, Suttorp-Schulten MS, Viergever MA, Russell SR, Van Ginneken B. Evaluation of a system for automatic detection of diabetic retinopathy from color fundus photographs in a large population of patients with diabetes. Diabetes Care 2008; 31 (2): 193-8

15. Abràmoff MD, Reinhardt JM, Russell SR, Folk JC, Mahajan VB, Niemeijer M, et al. Automated early detection of diabetic retinopathy. Ophthalmology 2010; 117 (6): 1147-54.

16. Scotland GS, McNamee P, Fleming AD, Goatman KA, Philip S, Prescott GJ, et al. Costs and consequences of automated algorithms versus manual grading for the detection of referable diabetic retinopathy; Scottish Diabetic Retinopathy Clinical Research Network. Br J Ophthalmol 2010; 94 (6): 712-9.

17. Scanlon PH, Malhotra R, Greenwood RH, Aldington SJ, Foy C, Flatman M, et al. Comparison of two reference standards in validating two field mydriatic digital photography as a method of screening for diabetic retinopathy. Br J Ophthalmol 2003; 87(10):1258-63.

18. Niemeijer M, Van Ginneken B, Cree MJ, Mizutani A, Quellec G, Sánchez CI, et al. Retinopathy online challenge: automatic detection of microaneurysms in digital color fundus photographs. IEEE Trans Med Imaging 2010; 29 (1): 185-95.

19. Teng T, Lefley M, Claremont D. Progress towards automated diabetic ocular screening: a review of image analysis and intelligent systems for diabetic retinopathy. Med Biol Eng Comput 2002; 40 (1): 2-13.

20. Olson JA, Strachan FM, Hipwell JH, Goatman KA, McHardy KC, Forrester JV, et al. A comparative evaluation of digital imaging, retinal photography and optometrist examination in screening for diabetic retinopathy. Diabet Med 2003; 20 (7): 528-34.

21. Philip S, Fleming A, Goatman K, Fonseca S, Mcnamee P,
Scotland G, et al. The efficacy of automated "disease/no disease" grading for diabetic retinopathy in a systematic screening programme. Br J Ophthalmol 2007; 91 (11): 1512-7.

22. Klein R, Klein BE, Moss SE, Davis MD, DeMets DL. The Wisconsin Epidemiologic Study of Diabetic Retinopathy III. Prevalence and risk of diabetic retinopathy when age at diagnosis is 30 or more years. Arch Ophthalmol 1984; 102: 527-32.

23. Abràmoff $\mathrm{MD}$, Folk $\mathrm{JC}$, Han $\mathrm{DP}$, Walker JD, Williams DF, Russell SR, et al. Automated analysis of retinal images for detection of referable diabetic retinopathy. JAMA Ophthalmol 2013; 131 (3): 351-7.

24. Fleming AD, Goatman KA, Philip S, Prescott GJ, Sharp PF, Olson JA. Automated grading for diabetic retinopathy: a large-scale audit using arbitration by clinical experts. Br J Ophthalmol 2010; 94 (12): 1606-10.

25. Larsen N, Godt J, Grunkin M, Lund-Andersen H, Larsen $\mathrm{M}$. Automated detection of diabetic retinopathy in a fundus photographic screening population. Invest Ophthalmol Vis Sci 2003; 44 (2): 767-71.

26. Walter T, Klein JC, Massin P, Erginay A. A contribution of image processing to the diagnosis of diabetic retinopathy-detection of exudates in color fundus images of the human retina. IEEE Trans Med Imaging 2002; 21 (10): 1236-43.

27. Taylor D, Fisher J, Jacob J, Tooke JE. The use of digital cameras in a mobile retinal screening environment. Diabet Med 1999; 16 (8): 680-6.

28. Boucher MC, Desroches G, Garcia-Salinas R, Kherani A, Maberley D, Olivier S, et al. Teleophthalmology screening for diabetic retinopathy through mobile imaging units within Canada. Can J Ophthalmol 2008; 43 (6): 658-68.

29. Coronado F, Galaz D. DART Inteligencia artificial para la prevención de la ceguera, Entrevista a Jose Tomás Arenas y Dra. María Eugenia Chadwick. Laboratorio de gobierno 2017. Disponible en: https://lab.gob.cl/ uploads/filer_public/65/eb/65ebdd72-7f95-487e-a98575520df3ecac/5_dart.pdf [Consultado el 1 de abril de 2018]. 